\section{Detailed analysis of the oligodendrocyte myelin glycoprotein gene in four patients with neurofibromatosis 1 and primary progressive multiple sclerosis}

\author{
M R Johnson, R E Ferner, M Bobrow, R A C Hughes
}

21. It is concluded that PPMS in patients with NF1 can occur without concurrent mutation of the OMgp gene. The glycine to aspartic acid polymorphic alteration at codon 21 is neither sufficient nor necessary for the development of PPMS.

(F Neurol Neurosurg Psychiatry 2000;68:643-646)

Keywords: primary progressive multiple sclerosis; neurofibromatosis 1; oligodendrocyte myelin glycoprotein

Neurofibromatosis 1 (NF1) is a common autosomal dominant disorder with a minimum prevalence of 1 in $5000 .{ }^{1}$ Neurological manifestations range from usually mild cognitive impairment and optic nerve and parenchymal gliomas to epilepsy and macrocephaly. ${ }^{2}{ }^{3} \mathrm{We}$ have reported the case histories of five patients, including two siblings, who have both NF 1 and primary progressive multiple sclerosis $(\mathrm{PPMS})^{4}$ and have since identified a further patient with both NF1 and PPMS. More recently, a systematic clinical review of 138 unselected adult patients with NF1 identified one patient with a slowly progressive spastic paraparesis and multiple high signal hyperintensities on T2 weighted MRI. ${ }^{5}$ A population based study of NF1 identified a further patient with both NF1 and multiple sclerosis in a series of 135 patients with NF1.

The gene for NF1 is located on chromosome 17q11.2, spans $350 \mathrm{~kb}$ of genomic DNA, and contains 60 exons. ${ }^{6}$ Three genes, EV12A, EV12B and oligodendrocyte myelin glycoprotein (OMgp), are embedded within intron 27b of the NF1 gene. ${ }^{7}$ Each of these genes is transcribed off the opposite strand to NF1. EV12A and EV12B are of unknown function. OMgp is a membrane glycoprotein that appears in the human CNS at the time of myelination. ${ }^{8}$ It can be detected immunohistochemically in CNS myelin and on the surface of cultured oligodendrocytes, as well as at the paronodal region of myelin in peripheral nerve. ${ }^{9}$ The glycoprotein is anchored to the outer leaflet of the myelin membrane through a glycosylphosphatidyl inositol lipid molecule, as is the cell surface form of the neural cell adhesion molecule N-CAM. ${ }^{10}$ In addition, a subpopula- 
tion of OMgp molecules from human brain contains the HNK-1 carbohydrate. ${ }^{8}$ The HNK-1 epitope is expressed on those CNS glycoproteins implicated in adhesion, such as N-CAM, L1, and MAG. ${ }^{11}$ Structurally therefore, OMgp has the potential to function as an adhesion molecule and could contribute to the interactions between the plasma membranes of oligodendrocytes and axons required for myelination or survival of myelinated axons.

This study addresses the specific hypothesis that PPMS in patients with NF1 results from concurrent mutation of the OMgp gene.

\section{Methods}

All patient samples were obtained with consent. Four unrelated patients with NF1 and PPMS were studied (UMDS1A, 5A, 15A, and 20A). The case histories of three of the four patients (UMDS 1A, 5A, and 20A) have been published elsewhere ${ }^{4}$ (UMDS 1A corresponds to case 3 , UMDS $5 \mathrm{~A}$ to case 1 and UMDS 20A to case 5). UMDS 15A (not reported elsewhere) was a 24 year old right handed single man diagnosed as having neurofibromatosis 1 on the basis of multiple dermal neurofibromas, a facial plexiform neurofibroma, and a family history of the disease. He had cognitive impairment and was unable to read or write. He presented with a 2 year history of progressive unsteadiness and stiffness in his walking. On neurological examination he had pale optic discs and an ataxic nystagmus on left lateral gaze. There was a moderate spastic paraparesis with bilateral limb incoordination in both arms and legs and a marked gait ataxia. Brain MRI disclosed multiple lesions in the periventricular region on $\mathrm{T} 2$ weighted images which were typical of demyelination. Examination of CSF disclosed intrathecal oligoclonal bands.

DNA ANALYSIS

Southern blot analysis was performed as previously described. ${ }^{12}$ cDNA was prepared from polymerase chain reaction (PCR) amplification of wild type genomic DNA: F-GGG TGT ATC TGT TTG TTT CTA GGC; R-GTC CAT ATC TAA CCA GTG ATT CG. Amplifications were perfomed in $50 \mu \mathrm{l}$ volumes containing $100 \mathrm{ng}$ DNA, $300 \mathrm{ng}$ of each primer, $1 \times$ Taq polymerase buffer $(67 \mathrm{mmol} / 1$ Tris HCL, pH 8.8, $16.6 \mathrm{mmol} / 1\left(\mathrm{NH}_{4}\right)_{2} \mathrm{SO}_{4}, 6.7$ $\mathrm{mmol} / \mathrm{l} \mathrm{MgCl}_{2}, 170 \mu \mathrm{g} / \mathrm{ml} \mathrm{BSA}, 10 \mathrm{mmol} / 1$ B-mercaptoethanol), $0.5 \mathrm{mmol} / 1 \mathrm{dNTPS}$, and 0.5 units Taq DNA polymerase (Amplitaq). Cycling conditions consisted of initial denaturation at $94^{\circ} \mathrm{C}$ followed by 30 cycles of denaturation at $93^{\circ} \mathrm{C}$ for 1 minute, $61^{\circ} \mathrm{C}$ for 0.5 minutes, and $72^{\circ} \mathrm{C}$ for 1 minute followed by a final extension of $72^{\circ} \mathrm{C}$ for 5 minutes.

Quantitative PCR was carried out as previously described. ${ }^{13}$ Exon $0 \mathrm{~F}$ of the Fanconi anaemia gene served as the control locus. ${ }^{14}$ The terminal portion of the OMgp coding sequence was amplified with the following primers: F-CTG CAA CTC TAA CTA TTC ATC TCC; R-GTC CAT ATC TAA CCA GTG ATT CG. Chemical cleavage of mismatch (CCM) was performed as previously described and included reverse labelling of mismatch probes. ${ }^{15}$ OMgp sequences were amplified in three overlapping $1.2 \mathrm{~kb}$ segments: segment $\mathrm{A}$ : F-TAA TCG TAT TTC AGT ACT TC/RCAC ATT GAA TTA CAT TAC TG, segment B: F-TAG GTA CTG TGT TGG TCT GG/R-CTT ACT GAA GTG GAT GAT GG, and segment C: F-TCT CAC ACA TCT TTA CCT GC/R-CGT AGC TGC CAT CCC ATG AA. PCR amplification was as above with annealing temperatures of $55^{\circ} \mathrm{C}$ (segment $\mathrm{A}$ ) and $61^{\circ} \mathrm{C}$ (segments $\mathrm{B}$ and $\mathrm{C}$ ).

\section{Results}

Polymerase chain reaction based mutation analysis such as CCM or direct sequencing can fail to detect mutations if the mutation is due to duplication of a locus, deletion, or major rearrangement. In general, mutation screens fail to exclude such possibilities before PCR based analysis with resultant errors in the assignment of mutation status. However, because CNS dysmyelinating disorders are known to occur as a result of deletion/duplication of genes encoding myelin proteins we considered it essential to exclude a major gene rearrangement before detailed PCR based analysis.

Gross integrity of the OMgp locus in the four patients was initially established with Southern blot analysis (results not shown). To demonstrate conclusively that the OMgp sequence was neither deleted nor duplicated quantitative PCR was used to determine gene dosage. The mean dosage quotient (DQ) and SD for double copy loci were calculated from normal controls. No significant differences were seen between the DQ values for the exon pairs in any of the patient samples, thus demonstrating normal copy number of the OMgp gene in all four patients (results not shown).

Having confirmed gross integrity as well as copy number of the OMgp locus we proceeded to a detailed mutation analysis using the method of CCM; CCM mutation detection approaches $100 \%{ }^{16}$ The entire OMgp coding, non-coding, and intronic sequence together with approximately $1 \mathrm{~kb}$ of $5^{\prime}$ and $3^{\prime}$ flanking sequence was screened. A single mismatch band was identified in segment $\mathrm{B}$ from two patients (UMDS 1A and 20A, data not shown). This corresponds to a $G$ to $A$ transition (GGT to GAT) resulting in an amino acid change of glycine to aspartic acid at codon 21 . This polymorphic change leads to loss of a BstN1 restriction site at this position (figure A) which was used to confirm the change at the level of genomic DNA (figure B). No other mismatch bands were detected.

\section{Discussion}

This work establishes that the clinical association of PPMS and NF1 cannot be explained simply in terms of concurrent mutation of the OMgp gene. Furthermore, the glycine to aspartic acid alteration at codon 21 is neither sufficient nor necessary for the development of PPMS. One possible explanation for the conjunction of these two disorders is the unfortunate coincidence of chance. The maximum prevalence of NF1 is in the region of 1 in 5000. The maximum prevalence of multiple 

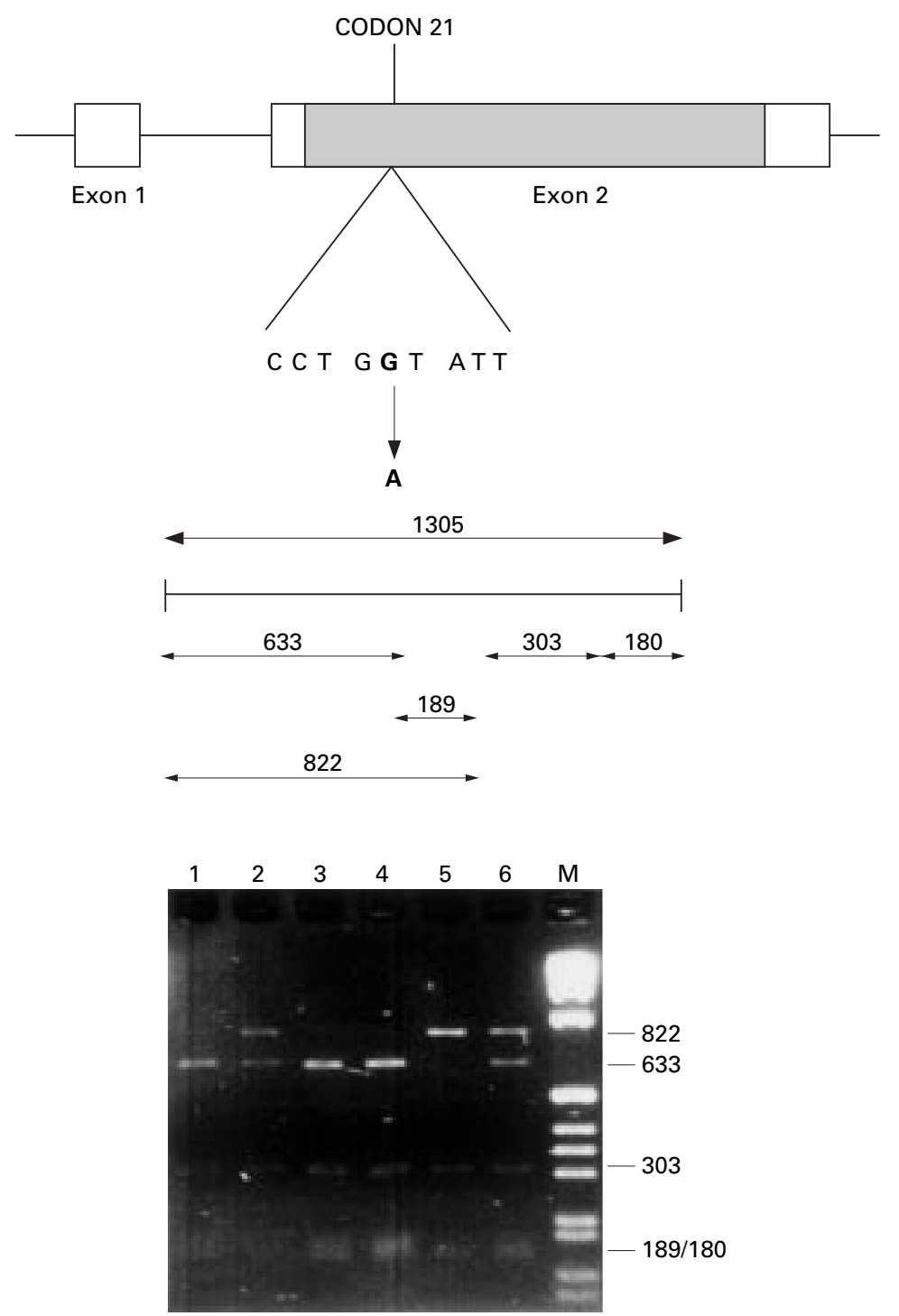

(A) BstN1 restriction map of amplified segment B (horizontal bar) from the OMgp locus. Open and hatched boxes as above. Horizontal arrows represent digestion products after $B s t N 1$ restriction of amplified segment B. Numbers given are in base pairs. Band 822 corresponds to the polymorphic allele. WT allele generates two fragments, 633 and $189 .(B)$ Agarose gel electrophoresis of segment B BstN1 digestion products. Lane1, control

homozygote WT; lane 2, UMDS 1 A (heterozygote WT / codon 21 polymorphism); lane 3, UMDS $5 A$ (homozygote WT); lane 4, UMDS 15 A (homozygote WT); lane 5, control homozygote codon 21 polymorphism; lane 6, UMDS $20 A$ (heterozygote WT/codon 21 polymorphism)

sclerosis in the United Kingdom is 1 in 900 of which primary progressive disease accounts for about $15 \%$. Using these figures, 14 patients in the United Kingdom with both multiple sclerosis and NF1 would be anticipated of which only two would be expected to have PPMS. To date, a total of six patients with NF1 and PPMS have been identified with a current prevalence of four (two of the six having died of unrelated disorders). Furthermore, it is likely that the six cases with PPMS and NF1 identified to date represent an under-ascertainment of cases as the diagnosis of NF1 often goes unnoticed unless carefully examined for by a specialist.

The aetiology of multiple sclerosis involves an interplay between a genetic predisposition and environmental triggers. ${ }^{17}{ }^{18}$ In addition to influencing disease susceptibility, genetic factors may also affect the clinical course. ${ }^{19}$ Little work has been done to explore the genetic mechanisms that might underlie the different clinical courses in multiple sclerosis. In a study of 87 sibling pairs with multiple sclerosis concordance for severity was found supporting the hypothesis that disability might be influenced by familial factors. ${ }^{20}$ By contrast, a separate study of 177 sibling pairs demonstrated a lack of concordance for disability, but strong correlation for disease course. ${ }^{21}$ Taken together, these results suggest that clinical features in multiple sclerosis may be modified by inherited factors.

Therefore, further possible explanations for the occurrence of PPMS with NF1 are that OMgp acts to modify the multiple sclerosis phenotype (either through polymorphic variation of the OMgp gene or because concurrent mutation of the NF1 gene disrupts normal OMgp gene regulation), or that the OMgp gene is in linkage disequilibrium with a disease modifying gene. In the United Kingdom whole genome screen for susceptibility loci a weak association was described between the NF1 locus and multiple sclerosis. ${ }^{22}$ However, this study did not differentiate between clinical subtypes of multiple sclerosis. If the demyelinated plaque of multiple sclerosis is in part the result of different pathogenetic mechanisms, then a genetic association between the OMgp locus and PPMS may well have been masked in the whole genome screens performed to date. Whether or not the genetic alteration in this study predisposes to a primary progressive clinical course can be addressed by a properly controlled association study which recognises issues of population admixture and statistical power $^{23}$ as well as the problem of ascertaining patients with clinically definite PPMS. ${ }^{24}$ In recruitment of patients with PPMS for a recent therapeutic trial, of 138 patients referred by experienced neurologists with a diagnosis of PPMS, 43 either did not have the disease or the diagnosis was not secure.

We are grateful to Dr P Kennedy for referring UMDS 20A and Dr M Jackson for the referring UMDS $15 \mathrm{~A}$. This work was funded by a grant from the Medical Research Council.

1. Huson SM, Harper PS, Compston DAS. Von Recklinghausen neurofibromatosis: a clinical and population study in south east Wales. Brain 1988;111:1355-81.

2 Ferner RE, Hughes RAC, Weinman J. Intellectual impairment in neurofibromatosis 1. f Neurol Sci 1996;138:125ment

3 Hughes RAC. Neurological complications. In: Huson SM, Hughes RAC, eds. The neurofibromatoses: a pathogenetic and clinical overview. London: Chapman and Hall, 1994:20453.

4 Ferner RE, Hughes RA, Johnson MR. Neurofibromatosis 1 and multiple sclerosis. $\mathcal{F}$ Neurol Neurosurg Psychiatry 1995; 58:582-5.

5 Creange A, Zeller J, Rostaing-Rigattieri S. et al. Neurological complications of neurofibromatosis type 1 in adulthood. Brain 1999;122:473-481.

6 Shen MH, Harper PS, Upadhyaya M. Molecular genetics of neurofibromatosis type 1 (NF1). F Med Genet 1996;33:117 .

$7 \mathrm{Li}$ Ying, O'Connell $\mathrm{P}$, Breidenbach $\mathrm{HH}$, et al. Genomic organisation of the neurofibromatosis 1 gene (NF1). Genomics 1995:25:9-18.

8 Mikol DD, Gulcher JR, Stefansson K. The oligodendrocyte myelin glycoprotein belongs to a distinct family of proteins 
and contains the HNK-1 carbohydrate. $f$ Cell Biol 1990;110:471-9.

9 Apostolski S, Sadig SA, Hays A, et al. Identification of Gal(beta 1-3) GalNAc bearing glycoproteins at the nodes of Ranvier in peripheral nerve. $\mathcal{F}$ Neuroscience Res 1994;38 134-41.

10 Jessel TM. Adhesion molecules and the hierarchy of neural development. Neuron 1988;1:3-13.

11 Quarles RH. Glycoproteins of myelin sheaths. $7 \mathrm{Mol} \mathrm{Neuro-}$ sci $1997 ; 8: 1-12$.

12 Sambrook J, Fritsch EF, Maniatis T. Molecular cloning: a laboratory manuel, 2nd ed. Cold Spring Harbor: Cold Spring Harbor Lab Press, 1989.

13 Yau SC, Bobrow M, Mathew CG, et al. Accurate diagnosis of carriers of deletions and duplications in Duchenne/ Becker muscular dystrophy by fluorescent dosage analysis. f Med Genet 1996;33:550-8.

14 Gibson RA, Buchwald M, Roberts RG, et al. Characterisation of he exon structure of the Fanconi anaemia group $\mathrm{C}$ tion of he exon structure of the Fanconi anaemia group

15 Naylor JA, et al. Detection of three novel mutations in two

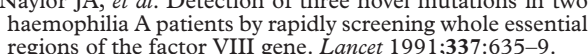

16 Roberts RG, Bobrow M, Bentley DR. Point mutations in the dystrophin gene. Proc Natl Acad Sci 1992;89:2331-5.

17 Ebers GC, Bulman DE, Sadovnick AD. A population-based study of multiple sclerosis in twins. $N$ Engl $f \mathrm{Med}$ 1986;315:1638-42.

18 Mumford CJ, et al. The British Isles survey of multiple sclerosis in twins. Neurology 1994;44:11-15.

19 Miller DH. Clinical and magnetic resonance imaging heterogeneity in multiple sclerosis. F Neurol Neurosurg Psychiatry 1999;67:139.

20 Brassat et al. Familial factors influence disability in MS multiplex families. Neurology 1999;52:1632-6.

21 Robertson NP et al. Clinical concordance in sibling pairs with multiple sclerosis. Neurology 1996;47:347-52.

22 Sawcer S, Jones HB, Feakes R, et al. A genome screen in multiple sclerosis reveals susceptibility loci on chromosome $6 \mathrm{p} 21$ and $17 \mathrm{q} 22$. Nature Genet 1996;13:464-468.

23 Todd JA. Interpretation of results from genetic studies of multifactorial diseases. Lancet 1999;345(suppl I):15-16.

24 Leary SM, Stevenson VL, Miller DH, et al. Problems in designing and recruiting to therapeutic trials in primary progressive multiple sclerosis. $\mathcal{F}$ Neurol 1999;246:562-8. 